Ophthalmologe 2022 $\cdot 119: 516-519$ https://doi.org/10.1007/s00347-021-01498-y Eingegangen: 11. Juni 2021 Überarbeitet: 18. August 2021

Angenommen: 23. August 2021

Online publiziert: 20 . September 2021

(c) Springer Medizin Verlag $\mathrm{GmbH}$, ein Teil von Springer Nature 2021

\section{Akute unilaterale Sehstörung nach COVID-Impfung}

\author{
P. Bachmann ${ }^{1}$ (D) C. Y. Mardin · A. J. Bartsch · J. M. Weller \\ ${ }^{1}$ Augenklinik, Universitätsklinikum Erlangen, Erlangen, Deutschland
}

\section{Anamnese}

Ein 78-jähriger pensionierter Zoologe stellte sich Mitte April in der internistischen Notaufnahme vor, weil er seit dem Vortag Einblutungen in der Haut des Gesichts und der Beine und im weiteren Verlauf $\mathrm{Na}$ sen- und Zahnfleischbluten sowie eine Hämaturie bemerkt habe. Außer den genann- ten Befunden äußerte der Patient subjektives Wohlbefinden ohne Infektzeichen oder B-Symptomatik. Relevante Vorerkrankungen bestanden keine, ebenso nahm der Patient keine regelmäßige Medikation ein. Ungefähr 3 Wochen vor der Erstvorstellung habe der Patient seine erste COVID19-Impfung mit Vaxzevria ${ }^{\circledR}$ - (AstraZeneca, Cambridge, Großbritannien) Impfstoff er-

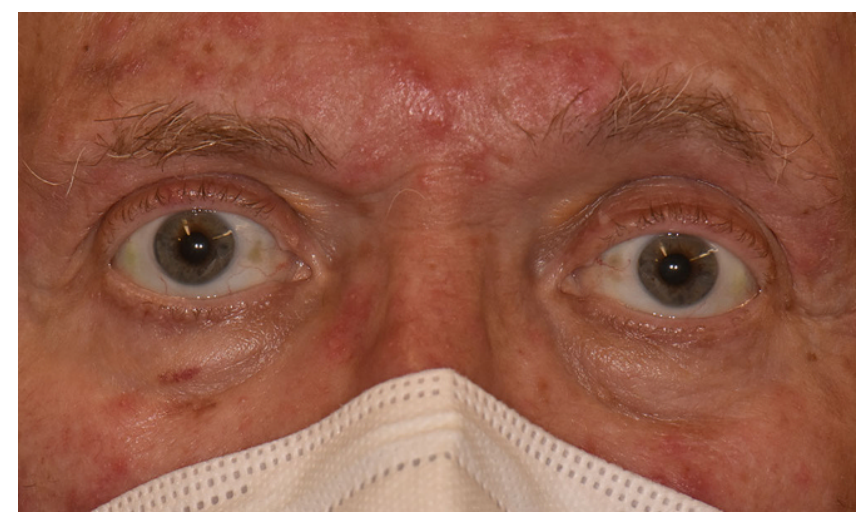

Abb. $1 \varangle$ Patient mit petechialen Einblutungen am Gesicht sowie an den Beinen (nicht auf dem Bild)

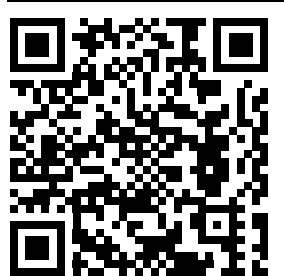

QR-Code scannen \& Beitrag online lesen
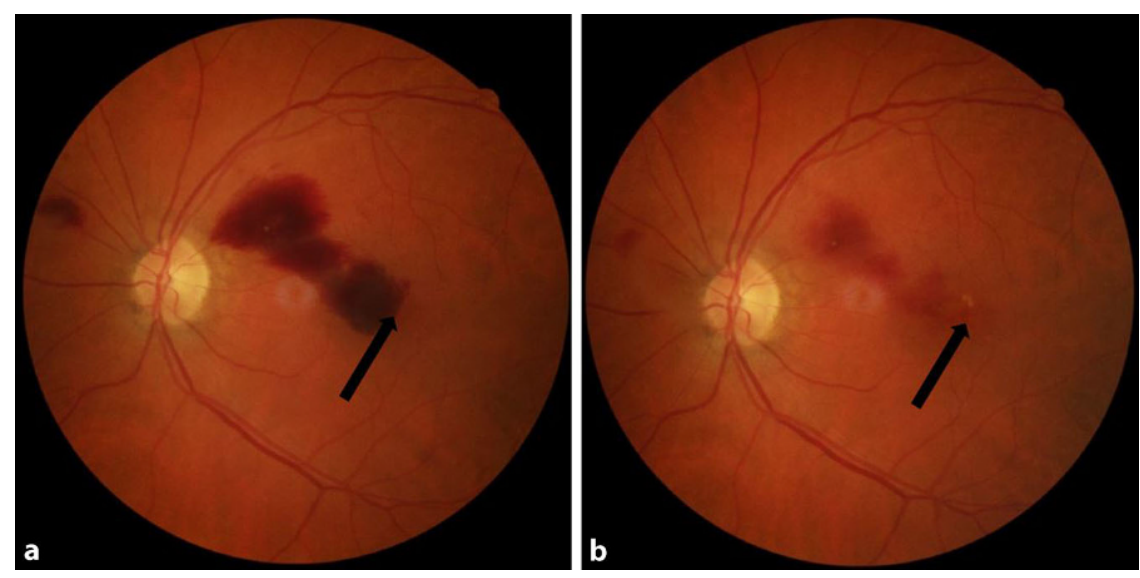

Abb. $2 \Delta$ Fundusfotografien des rechten Auges im zeitlichen Verlauf. Die Fovea ist mit einem Pfeil gekennzeichnet. a Initialer Befund. Intraretinale Blutungen, zum Teil fleckig und zum Teil streifig, peripapillär bis an die Fovea heranreichend. $\mathbf{b}$ Befund nach 4 Wochen. Langsame Resorption der intraretinalen Blutung mit Größenabnahme. Die Fovea ist weniger einbezogen als zu Beginn, was zu einem Visusanstieg und subjektiver Beschwerdebesserung führte 

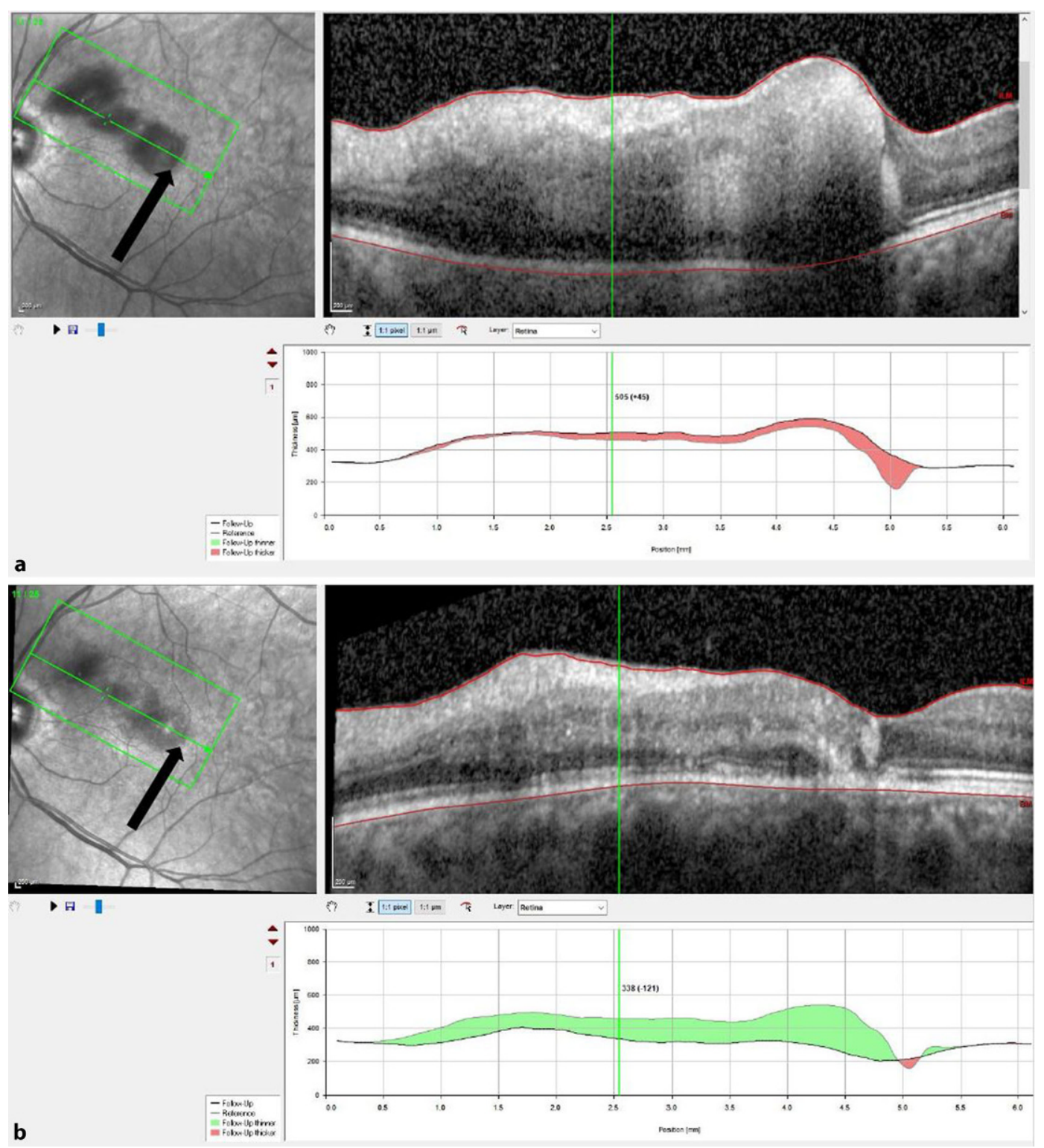

Abb. $3 \triangleleft$ Zwei SD-OCTAufnahmen des linken Auges im zeitlichen Verlauf, a Infrarotbild, b b-Scan, unten Dickeprofil (Spectralis HRA + OCT, Heidelberg Engineering, Heidelberg, Deutschland). Die Fovea ist mit einem Pfeil gekennzeichnet. a Diagonaler Rasterscan über Blutung, initialer Befund. Intraretinale Blutung, welche nahe Papille beginnt und schräg nach temporal inferior zieht unter partieller Einbeziehung der Fovea, welche das Rotsehen sowie den Visusverlust erklärt. b Diagonaler Rasterscan über Blutung, Befund nach 4 Wochen OCTAufnahme des linken Auges. In der Dickemessung der Netzhaut zeigt sich die intraretinale Blutung rückläufig, die Fovea ist weniger einbezogen als in der Voruntersuchung, was für den Patienten den subjektiven Rückgang der Metamorphopsien bedeutete. Aufgrund der Dickenverminderung und Irregularität der ellipsoiden Schicht und äußeren Körnerschicht ist eine Restitutio ad integrum nicht sicher gewährleistet halten. Im Verlauf des stationären Aufenthaltes erfolgte zunächst ein Konsil am Bett des Patienten, da eine internistische Überwachung aufgrund der Hämorrhagien ein Konsil in der Augenklinik nicht zuließ. Die ophthalmologische Untersuchung erfolgte aufgrund von akuten Sehstörungen des Patienten am linken Auge.

Im Verlauf erfolgte die konsiliarische Vorstellung des Patienten in der Augenklinik, da er am linken Auge einen roten Schleier sehe, der zeitgleich mit oben genannten Symptomen aufgetreten sei und sein Sehen beeinträchtige.

\section{Klinischer Befund}

In der zentralen Notaufnahme zeigte sich eine starke Thrombozytopenie (1000 Thrombozyten pro Mikroliter), im manuellen Differenzialblutbild zeigten sich keine relevanten Auffälligkeiten. Bei der initial am Bett des Patienten durchgeführten Fundusuntersuchung zeigten sich retinale Einblutungen am hinteren Pol. Eine weiterführende Diagnostik in der Augenklinik, sobald sich der Allgemeinzustand stabilisiert hatte, wurde empfohlen.

Bei der Vorstellung in der Ambulanz der Augenklinik zeigt sich ein Patient mit punktförmigen, petechialen Einblutungen im Gesicht (-Abb. 1). Der Visus betrug am rechten Auge 1,0 und am linken Auge 0,2. Der Intraokulardruck lag im Normbereich.
Der vordere Augenabschnitt zeigte sich reizfrei mit einer altersentsprechenden Cataracta corticonuclearis. Fundoskopisch zeigt sich das rechte Auge ohne Auffälligkeiten, am linken Auge zeigen sich fundoskopisch intraretinale Blutungen, die sich zum Teil fleckig und zum Teil streifig darstellten. Diese waren ausschließlich am hinteren Pol, peripapillär bis an die Fovea heranreichend, lokalisiert (- Abb. 2). In der durchgeführten optischen Kohärenztomographie(OCT)Untersuchung (Heidelberg Spectralis OCT) konnten die Blutungen in den oberflächlichen und mittleren Netzhautschichten dargestellt werden (• Abb. 3a). In der durchgeführten Echographie zeigt sich die Netzhaut allseits anliegend. 


\section{Weiterer Verlauf}

Aufgrund der starken Thrombozytopenie erfolgte durch die internistische Klinik eine systemische Dexamethason- sowie eine Immunglobulintherapie, unter der es zu einem Anstieg der Thrombozytenwerte auf 108.000 pro Mikroliter kam. Es erfolgte eine umfangreiche Diagnostik, u. a. ein CMRT und eine angiologische Sonographie, um Blutungs- bzw. thrombotische Ereignisse auszuschließen. Weiterhin erfolgten ergänzende hämatologische Untersuchungen, welche den Verdacht einer vakzininduzierten thrombotischen Thrombozytopenie nicht erhärten konnten. Trotzdem wurde dem Patienten von einer erneuten Impfung mit dem AstraZeneca-Impfstoff von internistischer Seite abgeraten, da ein kausaler Zusammenhang zwischen der Impfung und der Autoimmunthrombozytopenie nicht ausgeschlossen werden kann. Von den federführend behandelnden Hämatologen erfolgte während des stationären Aufenthaltes eine Meldung an das PaulEhrlich-Institut über eine mögliche Impfnebenwirkung. Im Zuge der Vorstellung des Patienten in unserer Ambulanz wurde eine Therapie mit Prednisolonacetat $\mathrm{Au}$ gentropfen 5-mal täglich gestartet, unter welcher es bei einer Nachkontrolle nach 7 Tagen zu einer langsamen Resorption des intraretinalen Blutes kam. In der OCT kann ein Rückgang der Blutung dargestellt werden (๑ Abb. 3b).

Der Visus des Patienten stieg bei der Nachkontrolle nach 4 Wochen aufgrund der Blutung nahe der Makula leicht an auf 0,3 , er gab weiterhin einen deutlichen Rückgang des parazentralen Skotoms an.

\section{Diskussion}

Die Autoimmunthrombozytopenie (ITP, auch als idiopathische thrombozytopenische Purpura bekannt) ist definiert als eine Thrombozytopenie (weniger als 100.000 Thrombozyten pro Mikroliter) mit der Anwesenheit von Petechien insbesondere an den Beinen, Blutungen der Schleimhäute von Mund und Nase, urogenitalen Blutungen sowie inadäquaten Blutungen bei leichten Traumata bei ansonsten gesunden Erwachsenen oder Kindern [1]. Andere Blutwerte wie Leukozyten oder das Hämoglobin sind nicht verändert. Ursache ist meist die Autoantikörperbildung (lgG) gegen den Glykoprotein-Illb/llla-Komplex, wodurch es letztendlich zu einem schnelleren Abbau der Thrombozyten v. a. in der Milz kommt. Beim Erwachsenen kommt die ITP meist im Alter um 60 Jahre vor, bei Kindern meist zwischen 2 und 5 Jahren. Von Bedeutung ist eine ausführliche Anamnese, um verschiedene andere Ursachen für die Blutungen auszuschließen. Bei Vorliegen der oben genannten Symptome erscheint das Einbeziehen weiterer Fachrichtungen sinnvoll, da möglicherweise ein lebensbedrohliches Krankheitsbild die okulären Symptome verursachen kann. Zur Ursachenabklärung sind die Abnahme eines Blutbildes (technische Artefakte sind auszuschließen), von Gerinnungsparametern und ein Blutausstrich durch einen erfahrenen Hämatologen unerlässlich. Die Therapie richtet sich nach den Empfehlungen der American Society of Hematology [2] und ist von vielen Faktoren abhängig, u. a. von der Thrombozytenzahl und der Schwere der Blutungen. Falls die Thrombozytenzahl noch mehr als 20.000-30.000 pro Mikroliter beträgt, kann ein abwartendes Verhalten mit regelmäßigen Kontrollen ("watch and wait") vertretbar sein. Alternativ können Kortikosteroide gegeben werden. Bei schweren Verläufen muss die gleichzeitige Gabe von Kortikosteroiden sowie Immunglobulinen erfolgen, ggf. mit weiteren Therapien, wie z.B. die Gabe von Thrombozytenkonzentraten oder Rituximab. Eine okuläre Beteiligung bei ITP ist möglich, jedoch extrem selten und manifestiert sich beispielsweise als Glaskörperblutung, Blutungen im Bereich des Tractus opticus, eine nicht arteriitische anteriore Optikusneuropathie oder auch eine subkonjunktivale Blutung [3]. Eine isolierte Thrombozytopenie sorgt im Regelfall nicht für eine retinale Blutung, stellt in Kombination mit anderen Komorbiditäten wie beispielsweise einer diabetischen Retinopathie jedoch wahrscheinlich einen Risikofaktor dar. In der aktuellen Literatur sind bei bisher gesunden Patienten nur wenige Fälle einer intraretinalen Blutung bei ITP [3-5].

Von der ITP abzugrenzen ist das Erkrankungsbild der prothrombotischen Immunthrombozytopenie, die mit Sinusvenenthrombosen oder einer disseminierten intravasalen Koagulopathie (DIC) einhergehen kann und durch Impfungen getriggert werden kann.

In In-vitro-Analysen konnte gezeigt werden, dass topische verabreichte Steroide eine Glaskörperpenetration zeigen, zur Wirksamkeit von Prednisolon-Augentropfen bei intraretinalen Blutungen gibt es jedoch bisher kaum Literatur, weshalb die Gabe bei diesem Patienten nicht evidenzbasiert erfolgt ist. Eine operative Therapie mittels rTPA-/Gasinstillation wurde von uns in Betracht gezogen. Die Wirkung von rtPA und Gas bei subretinalen Blutungen besteht auf dem Prinzip der Verdrängung der Blutung aus der $\mathrm{Ma}$ kula. Ein solcher verdrängender Effekt ist jedoch bei intraretinalen Blutungen nicht zu erwarten. Angesichts der bei unserem Patienten schon länger zurückliegenden Blutung und fehlender Evidenz haben wir bei fraglicher Verbesserung des Visus durch eine operative Intervention von einer rTPA-/Gasinstillation abgesehen.

Angesichts der COVID-19-Pandemie wurde in den letzten Monaten ein besonderer Fokus der medizinischen Forschung auf eine Impfung gegen das Virus gelegt, die verschiedenen Präparate sind mittlerweile hinlänglich bekannt. Der im Fall gezeigte Patient wurde mit dem Adenovirus-Vektor-Impfstoff Vaxzevria ${ }^{\circledR}$ von AstraZeneca geimpft. Im Rahmen der Impfung mit diesem Präparat wurden mehrere thrombotische Ereignisse sowie Fälle einer prothrombotischen Immunthrombozytopenie beobachtet, jedoch bisher keine Fälle einer mutmaßlich durch die Impfung getriggerten Immunthrombozytopenie. In der EMA-Bewertung des Impfstoffs sind keine okulären Nebenwirkungen angegeben (www.ema.europa. eu/). Ein kausaler Zusammenhang zwischen der erfolgten Impfung und der aufgetretenen ITP lässt sich nicht beweisen. Eine Meldung an das Paul-EhrlichInstitut ist im Falle des Verdachtes einer 
Nebenwirkung auf die COVID-Impfung möglich. Genaue Richtlinien, $a b$ wann von einer Impfnebenwirkung auszugehen ist, gibt es nicht.

\section{》) Diagnose: Intraretinale Blutungen im Rahmen einer Autoimmunthrombozytopenie}

Aufgrund der vielfältigen Manifestationen von COVID-19 und der möglichen Nebenwirkungen nach der Impfung gegen das Virus erscheint es aus ophthalmologischer Sicht sinnvoll, bei Vorliegen einer intraokularen Blutung ohne plausible Erklärung und kürzlich erfolgter Impfung den Patienten interdisziplinär zu diagnostizieren und zu therapieren.

\section{Fazit für die Praxis}

- Bei ein- bzw. beidseitiger akuter Visusminderung ist insbesondere in Zeiten von COVID-19 eine genaue Anamnese wichtig, um eine COVID-Erkrankung bzw. -Impfung und Hinweise auf deren möglicherweise lebensbedrohliche systemische Komplikationen zu erfahren.

- Im Falle einer ITP kann es auch bei bisher völlig gesunden Patienten zu intraretinalen Blutungen kommen.

- Bei Verdacht auf eine Augenbeteiligung bei akuter systemischer Erkrankung ist es wichtig, zeitnah andere Fachdisziplinen zu involvieren und schnell weitere Diagnostik zu planen.

Korrespondenzadresse

\section{P. Bachmann, M.D.}

Augenklinik, Universitätsklinikum Erlangen

Erlangen, Deutschland

Philipp.Bachmann@uk-erlangen.de

\section{Einhaltung ethischer Richtlinien}

Interessenkonflikt. P. Bachmann, C.Y. Mardin, A.J. Bartsch und J.M. Weller geben an, dass kein Interessenkonflikt besteht.

Für diesen Beitrag wurden von den Autoren keine Studien an Menschen oder Tieren durchgeführt. Für die aufgeführten Studien gelten die jeweils dort angegebenen ethischen Richtlinien. Für Bildmaterial oder anderweitige Angaben innerhalb des Manuskripts, über die Patienten zu identifizieren sind, liegt von innen und/oder ihren gesetzlichen Vertretern eine schriftliche Einwilligung vor.

\section{Literatur}

1. Rodeghiero F, Stasi R, GernsheimerT, Michel M, Provan D, Arnold DM, Bussel JB, Cines DB, Chong BH, Cooper N, Godeau B, Lechner K, Mazzucconi MG, McMillan R, Sanz MA, Imbach P, Blanchette V, Kühne T, Ruggeri M, George JN (2009) Standardization of terminology, definitions and outcome criteria in immune thrombocytopenic purpura of adults and children: report from an international working group. Blood 113(11):2386-2393

2. Neunert C, Terrell DR, Arnold DM, Buchanan G, Cines DB, Cooper N, Cuker A, Despotovic JM, George JN, Grace RF, Kühne T, Kuter DJ, Lim W, McCrae KR, Pruitt B, Shimanek H, Vesely SK (2019) American Society of Hematology 2019 guidelines for immune thrombocytopenia. Blood Adv3(23):3829-3866

3. Goel N, Arora S, Jain P, Ghosh B (2014) Massive subretinal and vitreous haemorrhages at presentation in idiopathic thrombocytopenic purpura: report of a case and review of literature. Clin Exp Optom 97(3):270-273. https://doi.org/10.1111/ cxo. 12072

4. Majji AB, Bhatia K, Mathai A (2010) Spontaneous bilateral peripapillary, subhyaloid and vitreous hemorrhage with severe anemia secondary to idiopathic thrombocytopenic purpura. Indian J Ophthalmol 58(3):234-236. https://doi.org/10. 4103/0301-4738.62651

5. Tiede A, Sachs UJ, Czwalinna A, Werwitzke $S$, Bikker R, Krauss JK, Donnerstag FG, Weißenborn K, Höglinger GU, Maasoumy B, Wedemeyer $H$, Ganser A (2021) Prothrombotic immune thrombocytopenia after COVID-19 vaccine. Blood. https:// doi.org/10.1182/blood.2021011958
DOG

Deutsche Ophthalmologische Gesellschaft

Gesellschaft

für Augenheilkunde

\section{Bereich für Mitglieder der DOG auf SpringerMedizin.de}

Schauen Sie sich den neuen Bereich für Mitglieder der DOG auf SpringerMedizin.de an: www.springermedizin.de/dog

Hier finden Sie

- die Leitlinien, Empfehlungen und Stellungnahmen der DOG auf einen Blick,

- schnellen Zugriff auf die CME-Kurse von Der Ophthalmologe,

- das Online Archiv von Der Ophthalmologe rückwirkend bis 1997,

- die aktuelle Ausgabe als e.Paper zum Offline-Lesen unterwegs,

- direkte Links zur Webseite der DOG und zum DOG E-Campus.

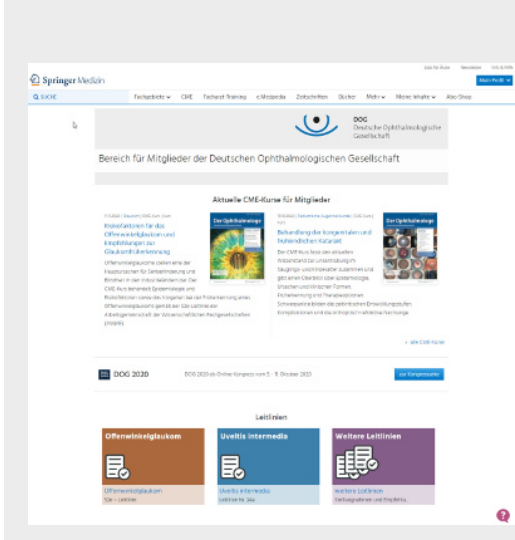

Registrieren Sie sich einmal kostenfrei auf www.springermedizin.de. Geben Sie dabei Ihren Vor- und Nachname und Lieferadresse wie auf dem Adressaufkleber auf Ihrem Heft an. So kann im System die Zugehörigkeit zur DOG sichergestellt werden.

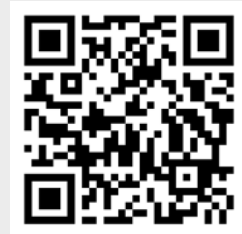

Einscannen und schnell und einfach lossurfen 